\title{
Comme un cri, un feu ou un animal qu'on ne peut étouffer
}

Os Verdes Anos (Les Vertes années, 1963) et Mudar de Vida (Changer de vie, 1966) de Paulo Rocha

Like a Scream, a Fire or an Animal that Cannot Be Smothered. Paulo Rocha's

Os Verdes Anos (1963) \& Mudar de Vida (1966)

\section{Guillaume Bourgois}

\section{OpenEdition}

Journals

Édition électronique

URL : http://journals.openedition.org/recherchestravaux/1041

DOI : 10.4000/recherchestravaux.1041

ISSN : 1969-6434

Éditeur

UGA Éditions/Université Grenoble Alpes

Édition imprimée

ISBN : 978-2-37747-065-5

ISSN : 0151-1874

Référence électronique

Guillaume Bourgois, "Comme un cri, un feu ou un animal qu'on ne peut étouffer », Recherches \& Travaux [En ligne], 93 | 2018, mis en ligne le 26 octobre 2018, consulté le 08 septembre 2020. URL : http://journals.openedition.org/recherchestravaux/1041; DOl : https://doi.org/10.4000/ recherchestravaux.1041

Ce document a été généré automatiquement le 8 septembre 2020

(C) Recherches \& Travaux 


\section{Comme un cri, un feu ou un animal qu'on ne peut étouffer}

Os Verdes Anos (Les Vertes années, 1963) et Mudar de Vida (Changer de vie, 1966) de Paulo Rocha

Like a Scream, a Fire or an Animal that Cannot Be Smothered. Paulo Rocha's

Os Verdes Anos (1963) \& Mudar de Vida (1966)

\section{Guillaume Bourgois}

1 Parlant de César, dont la stature immense confisque l'Histoire, la renommée et la gloire, le Cassius de Shakespeare déclare à Brutus, c'est-à-dire à celui qui associera son nom à la fois à une forme de régicide et à une forme de parricide :

Mais, mon ami, c'est qu'il chevauche ce monde étroit

Comme un Colosse, et que nous autres nabots

Marchons sous ses jambes géantes, furetant

Pour nous trouver des tombes sans honneur ${ }^{1}$.

2 Le moins que l'on puisse dire, c'est que Paulo Rocha a toujours revendiqué une attache affective et esthétique à d'autres cinéastes ayant voulu (et su) s'intéresser et donner la parole aux oubliés des discours et représentations officielles, à ceux qu'occulte l'Histoire, condamnant leurs actes et leurs modes de vie aux tombes déshonorantes et à l'infamie pensée par Michel Foucault.

Rocha a fait ses débuts en tant que collaborateur de Manoel de Oliveira, figure pour lui de mentor et d'expert en irrévérence - irrévérence qui l'amènera par exemple à tourner au début des années quatre-vingt-dix son chef-d'œuvre historique en forme de contre-Histoire, Non ou la vaine gloire de commander (Non ou a vã glória de mandar, 1990), portant uniquement sur les défaites du Portugal et suivant principalement les existences de soldats et marins absents des ouvrages de référence. Rocha a notamment participé à Acte du printemps (Acto da primavera, 1963) et La Chasse (A Caça, 1964), deux films qui cherchent à pourfendre les clichés folklorisants en prenant appui sur la puissance barbare des paysages, de la langue et des coutumes populaires du nord du pays. 
Via ce compagnonnage intellectuel et artistique avec Oliveira, qui prend en 1992 la forme d'un documentaire qu'il lui consacre et intitule Oliveira, l'architecte, Rocha place aussi son œuvre dans le prolongement de celle de Camilo Castelo Branco, écrivain romantique du xix siècle, référence littéraire obsessionnelle à la fois chez Oliveira et João César Monteiro, et pour qui l'infamie fut une question centrale, une question personnelle, une question de famille et une question artistique. Incarcéré pour adultère et déshonneur, Castelo Branco mit à profit son séjour en prison pour effectuer un double geste foucaldien : il s'inspira du document officiel condamnant à l'exil l'un de ses ancêtres ayant commis un crime passionnel, document prétendument retrouvé dans des archives, pour composer le récit de son livre le plus connu, Amour de perdition, et tint en parallèle un journal publié de façon posthume sous le titre Memórias do cárcere ("Mémoires du cachot») dans lequel il consigna les récits des vies minuscules, violentes et hors-la-loi de ses compagnons d'enfermement et d'infortune.

Rocha a en outre collaboré avec António Reis, poète et cinéaste dont l'une des œuvres les plus importantes, le moyen-métrage Jaime de 1974, est tributaire de Foucault et de son Histoire de la folie à l'âge classique. S'intéressant à la vie, aux tableaux, dessins et textes de Jaime Fernandes, patient de l'hôpital Miguel Bombarda diagnostiqué schizophrène et réduit au statut infâmant de "cas clinique ", le film constitue une diatribe contre la psychiatrie institutionnelle en tant que lieu emblématique de la société de contrôle : les panoramiques circulaires soulignent le dispositif panoptique de l'hôpital et un extrait off de Saint James Infirmary, interprété par Louis Armstrong et consacré à une léproserie londonienne, retrace en reprenant Foucault une histoire des lieux d'enfermement de la société occidentale. De Pedro Costa à João Pedro Rodrigues, les cinéastes contemporains n'ont de cesse, à longueur d'interviews et dans leurs œuvres où Vanda, Ventura, Tónia ou Sérgio se font les porte-paroles et les porte-cris de tous les marginaux du pays, de rappeler tout ce qu'ils doivent à Reis et Rocha - et de donner ainsi à penser une histoire du cinéma moderne portugais qui confond ses traits avec une certaine histoire de l'infamie.

6 Au-delà de ce dialogue constant entre ses films et ceux des autres grands noms du cinéma portugais, qui a fait de ses œuvres le lieu idéal de cristallisation des enjeux modernes et transformé Rocha dès ses débuts en figure de proue du Cinema Novo - mouvement considéré comme le parent brésilien et portugais du Néoréalisme et de la Nouvelle Vague -, la filmographie du cinéaste se singularise par un tropisme japonisant qui prendra chaque fois plus d'importance. Rocha fut notamment attaché culturel à Tokyo entre 1975 et 1983, ce qui lui permit d'étudier la vie du diplomate portugais Wenceslau de Moraes (1854-1929) qui s'installa au Japon et adopta les coutumes du pays - personnage haut en couleur au cœur de plusieurs de ses œuvres aux allures d'autoportraits, dont L'île des amours (A Ilha dos amores, 1982). Au contact de la culture et du cinéma japonais, qui poussent son esthétique à l'hybridation et au choc, Rocha s'imprègne de films profondément révoltés, voire libertaires, qui eux aussi ne se sont imposés d'autre mission que celle de révéler les trajets des hommes et femmes de mauvaise vie peuplant aussi bien les chefs-d'œuvre de Mizoguchi, Les Contes de la lune vague après la pluie (Ugetsu monogatari, 1953) et La Rue de la honte (Akasen chitai, 1956) en tête, que les formes excessives du pinku eiga ${ }^{2}$.

7 Comme pour résumer une carrière qui s'est toute entière construite autour d'une frontière fictive entre le Portugal et le Japon, Paulo Rocha a réalisé deux documentaires pour la série "Cinéastes de notre temps »: Oliveira, l'architecte mais aussi trois ans plus 
tard un portrait tonitruant d'Imamura intitulé Shohei Imamura, le libre penseur (1995). Avec une sublime et mélancolique dernière séquence, où l'on voit le cinéaste japonais se promener entre les tombes déshonorantes de prostituées que la société a sacrifiées, le réalisateur portugais donne à ressentir la nécessité bouleversante qui a été et continue d'être celle de son cinéma, et celle de ceux dont le travail compte pour lui, de raconter les vies des hommes et des femmes infâmes pour mieux ne jamais faire taire leurs hurlements.

Rien d'étonnant ainsi à ce que les deux premiers longs métrages de Paulo Rocha, Les Vertes années (1963) et Changer de vie (1966), abordent la question de l'infamie foucaldienne - bien qu'ils ne respectent pas la première règle mentionnée par le philosophe dans la préface au recueil de vies qu'il projetait de constituer («qu'il s'agisse de personnages ayant existé réellement $\left.{ }^{3} »\right)$ puisqu'ils ne font que s'inspirer d'histoires liées aux milieux qu'ils explorent. Les deux films forment un diptyque, marqué par l'utilisation de morceaux originaux composés et joués à la guitare portugaise par Carlos Paredes et par la présence d'une même actrice, Isabel Ruth, et ils envisagent la problématique de façon complémentaire.

Les Vertes années se passe à Lisbonne et l'on suit une histoire de vie infâme au sens strict, à travers le trajet d'un jeune cordonnier que les circonstances, un amour blessé et une société suffoquant sous le poids d'un présent sans issue transformeront en meurtrier; en assassinant sa compagne, il assassinera tout espoir. La naïveté du personnage, couplée à la maladresse presque bressonienne du jeu de son interprète, la ligne aussi claire que mystérieuse que dessine son existence, du moment de l'arrivée de la figure centrale dans la capitale - où déjà le jeune homme se perd dans la grisaille - à cette nuit où le personnage est saisi par la fureur et rejoint, en essuyant sa main ensanglantée sur l'épaule d'un serveur, le Peter Lorre de $M$ le maudit (M, 1931), tout concourt à la mise en place d'un équilibre instable voulu par Foucault :

J'ai voulu [...] que ces personnages soient eux-mêmes obscurs; que rien ne les ait prédisposé pour un éclat quelconque, qu'ils n'aient été dotés d'aucune de ces grandeurs qui sont établies et reconnues [...], qu'il y ait dans leurs malheurs, dans leurs passions, dans ces amours et ces haines quelque chose de gris et d'ordinaire au regard de ce qu'on estime d'habitude digne d'être raconté ; que pourtant ils aient été traversés d'une certaine ardeur, qu'ils aient été animés par une violence, une énergie, un excès [...] qui leur donnent aux yeux de leur entourage, et à proportion de sa médiocrité même, une sorte de grandeur effrayante ou pitoyable ${ }^{4}$.

Délaissant la grisaille lisboète pour embrasser les violents effets de contraste permis par les paysages et la sublime luminosité d'un village de pêcheur du Nord du Portugal, Changer de vie re-problématise la question foucaldienne. Grâce au récit du retour chez lui d'un homme ayant passé plusieurs années à se battre dans les colonies après avoir été enrôlé de force, et à son histoire sentimentale avec une énigmatique jeune femme que tous méprisent en raison de son immense appétit de liberté, le film explore la façon dont une société frappe du sceau d'une infamie aux connotations sexuelles tous ceux qui se font les porteurs d'un discours, d'un comportement ou d'une réalité qu'elle ne veut pas entendre. Afin de confiner au hors champ, au non dit et donc de déréaliser la guerre coloniale, les personnages que croisent la figure centrale associent sa campagne militaire à une forme de tourisme sexuel, suggérant qu'il a passé autant de temps en Afrique uniquement pour pouvoir s'adonner à des orgies avec les autochtones ${ }^{5}$. Ils réduisent en parallèle les souffrances existentielles de sa compagne, dont on apprend qu'elle a tenté de mettre fin à ses jours par le passé, aux conséquences d'une vie de 
débauche et vont jusqu'à imaginer dans leurs tristes fantasmes que sa tentative de suicide correspond à un avortement déguisé.

11 Le second long-métrage de Rocha joue en permanence sur les effets d'attente et de suspense suscités par la tension de ses situations. Un couteau brandi, une parole malheureuse, un ton qui monte, des coups de fusil au loin, une foule d'éléments paraissent a priori des indices du surgissement d'une scène tragique qui enfermera définitivement les deux figures principales dans des existences vouées au malheur et à l'emprisonnement - «Ça va mal finir » est-il dit à plusieurs reprises. Ces menaces sont toutefois rapidement désamorcées, évitant ainsi l'issue fatale et violente à laquelle mène la plupart du temps la vie infâme, celle que connaît l'existence du jeune cordonnier des Vertes années, sans que cela anéantisse la dimension contestataire de leur excès foucaldien, bien au contraire. Adelino et Albertina, les deux personnages principaux, détruisent les clichés et mensonges de leur entourage, à la racine d'une honte infâme qu'on cherche à leur imposer, pour mieux choisir un comportement dérangeant et sacrilège (la jeune femme vole l'argent de la quête dans une chapelle, puis dépouille son propre frère, son amant ne cesse de brutaliser verbalement ses proches afin de dénoncer leur hypocrisie) et faire souffler le vent de révolte qui anime l'infamie. Adelino est fréquemment associé à la figure du loup, notamment lorsque ses appels off dans la forêt font peur à une Maria Barroso habillée comme le Chaperon Rouge ou lorsque retentissent les armes des chasseurs, et Albertina à un oiseau rebelle qui, quand elle se cache dans un puits, est trahie par l'envol de plusieurs oiseaux - comme si leur union parvenait à marier deux symboles d'une même soif de liberté, le «cane senza padrone» de Pasolini et la fière Carmen de Bizet. Cette soif de liberté et d'indépendance trouve une forme d'accomplissement dans leur décision finale d'affronter l'avenir aux côtés l'un de l'autre.

12 La référence au Petit Chaperon Rouge n'est pas anodine. Foucault revendique la « nouvelle ${ }^{6}$ » littéraire comme modèle narratif pour le récit de ses vies infâmes et les deux premiers films de Rocha reprennent ce modèle pour presque l'hystériser. La ligne claire du récit des Vertes années tient de la concision et de l'efficacité de la forme brève, et la construction de Changer de vie fait se succéder trois blocs d'une demi-heure chacun, presque indépendants les uns des autres, comme on adapterait trois nouvelles - Albertina n'intervient par exemple qu'au bout d'une heure et lance un nouveau mouvement. Fidèle à son tropisme japonisant, qui le pousse à filmer des marais comme on filmerait des rizières, à suggérer la présence de fantômes d'ancêtres dans les bois et à inscrire sans cesse les existences de ses personnages dans des cycles naturels, Rocha lorgne aussi du côté du conte, occidental mais surtout nippon, et compose un recueil filmique de contes de l'infamie à la manière du Mizoguchi des Contes de la lune vague après la pluie.

13 Ce qui est passionnant dans le texte de Foucault, c'est la fascination qu'exprime le philosophe pour la beauté étrange de la langue utilisée dans les lettres de cachet à partir desquelles il entend travailler :

Chacune de ces petites histoires de tous les jours devait être dite avec l'emphase de rares événements qui sont dignes de retenir l'attention des monarques; la grande rhétorique devait habiller ces affaires de rien ${ }^{7}$.

Mais le philosophe note également :

Il arrivait que les demandes d'internement soient faites par des gens de très petite condition, pas ou peu alphabétisés ; [...] [ils] composaient comme ils le pouvaient les formules et tours de phrase qu'ils pensaient requis lorsqu'on s'adressait au roi et 
aux grands, et ils les mélangeaient avec les mots maladroits et violents, les expressions de rustre [...]; au langage obligatoire et rituel s'entrelacent les rancœurs, les révoltes. Une vibration et des intensités sauvages bousculent les règles de ce discours guindé ${ }^{[}[. .$.$] .$

Par l'intervention fréquente des compositions de Carlos Paredes over, qui impriment aux séquences et aux dialogues leur rythme, portent la simplicité du regard filmique dans certaines scènes ou la complexité des mouvements de caméra utilisés dans d'autres, les deux films s'inscrivent volontairement dans l'héritage du fado, dont ils reprennent l'hybridité linguistique. Le fado mêle les textes des plus grands poètes et les expressions populaires, toujours au service de récits de "poèmes vies ${ }^{9}$ " évoquant les souffrances du peuple - contrairement à ce qu'a voulu faire croire le régime salazariste et sa propagande qui l'instrumentalisèrent au nom d'une défense nationaliste des valeurs soi-disant typiquement portugaises. Dans le même temps, le dispositif des Vertes années repose sur l'utilisation d'un narrateur en voix off, l'oncle de la figure centrale qui, tel les rédacteurs des lettres de cachet, mélange tournures savantes et formules d'argot afin de retracer la descente aux enfers de son neveu et prendre sa défense avant son enfermement définitif. Le scénario de Changer de vie a été écrit par le poète António Reis, pourfendeur du lyrisme artificiel de la poésie romantique et dont les textes célèbrent la beauté du quotidien et de la langue du peuple ${ }^{10}$. Dans leurs modalités de figuration et leurs répliques, les personnages modestes, principalement les pêcheurs de Changer de vie mais aussi les cordonniers des Vertes années, deviennent de sublimes poètes du quotidien - à l'image de ce couple de vieillards du film de 1966, dont la chambre aux murs fragiles semble transformée en temple ou en palais, et qui, malgré leur abattement, célèbrent la vie et la splendeur des étoiles.

Ce qui frappe surtout Foucault dans les lettres de cachet, c'est qu'elles obéissent à un art puissant et subtil de la disparate: disparate « entre les choses racontées et la manière de les dire ", " entre ceux qui se plaignent et supplient et ceux qui ont sur eux tout pouvoir ", "entre l'ordre minuscule des problèmes soulevés et l'énormité du pouvoir mis en œuvre ", " entre le langage de la cérémonie et du pouvoir et celui des fureurs et impuissances ${ }^{11}$ ». Or dans les deux films, la disparité langagière grâce à laquelle le parler du peuple est à la fois poème et fado se double d'une profonde disparité spatiale.

17 Non seulement la figure centrale des Vertes années donne souvent l'impression d'évoluer dans un décor qui ne semble pas vraiment à sa taille - Júlio est trop grand pour la chaise qu'on lui présente, il travaille dans un atelier trop petit pour lui, atelier se trouvant situé dans un entresol bâtard comme en signe d'exclusion de l'organisation sociale verticale lisboète, et on le voit perpétuellement écrasé par le gigantisme délirant des lieux de pouvoir construits par la dictature - mais l'espace de la capitale lui-même est violemment fragmenté. Les ellipses produisent à l'intérieur des séquences des sauts spatiaux aux allures de faux raccords entre des lieux très différents, et le film souligne l'hétérogénéité entre les vieux quartiers de Lisbonne, ses espaces modernes et urbanisés, et sa banlieue agricole où vivent la plupart des personnages. Au gré d'une visite guidée improvisée à travers la ville, l'oncle de Júlio tentera de réintroduire de l'unité et de la complémentarité entre les espaces dans l'esprit de son neveu; il échouera toutefois à véritablement ordonner la déraison spatiale.

L'effet de disparate est encore davantage marqué dans Changer de vie et ses implications politiques rendues plus claires. Le territoire hybride et sauvage qu'habitent les pêcheurs, composé de bois ténébreux, d'une plage laissant apercevoir une mer à la 
lumière aveuglante ou encore d'un marais énigmatique, s'oppose aux espaces froids, cliniques et sinistrement identiques de la société de contrôle salazariste, dans lesquels on cherche à dompter et dresser les corps populaires : l'hôpital, l'usine, et surtout un bureau pour l'emploi où Adelino et ses camarades sont soumis à des tests abêtissants dignes de ceux que l'on pourrait faire passer à des enfants de trois ans.

Dans ses deux premières œuvres, Rocha se fait le théoricien implicite du Cinema Novo et dresse une certaine cartographie de la modernité cinématographique par un système de clins d'œil et d'hommages : un style qui rappelle aussi bien le Néoréalisme que la Nouvelle Vague tout en évoquant parfois Mizoguchi, des scènes en écho à $M$ le maudit (Les Vertes années) ou aux Amants de la nuit (They Live By Night, 1949) de Nicholas Ray (Changer de vie), un salut amical à Glauber Rocha et au Cinema Novo brésilien, car l'interprète principal du second long-métrage est un acteur brésilien qui venait de tourner avec le futur réalisateur d'António Das Mortes (1969). À travers leur dynamique spatiale et l'hétérogénéité des influences revendiquées, ne serait-ce que pour ce qui est de leurs nationalités, Les Vertes années et Changer de vie font de la disparate foucaldienne l'arme esthétique et politique privilégiée de la modernité cinématographique. Par ce travail, Rocha annonce et complète par avance Deleuze. Analysant les effets de disjonction chez Foucault à la lumière de ceux des cinéastes modernes - notamment Duras, Syberberg et les Straub -, le philosophe en vient en effet à conclure que "Foucault est singulièrement proche du cinéma contemporain ${ }^{12}$."

Les deux films envisagent en parallèle la disparate dans une perspective goyesque. Foucault écrit dans L'Histoire de la folie à l'âge classique :

Les formes de Goya naissent de rien : elles sont sans fond, en ce double sens qu'elle ne se détachent que de la monotonie des nuits, et que rien ne peut assigner leur origine, leur terme et leur nature. Les Disparates sont sans paysages, sans murs, sans décor [...]. Mais dans cette nuit l'homme communique avec ce qu'il y a de plus profond en lui, et de plus solitaire ${ }^{13}$.

21 À l'issue d'un rendez-vous décevant entre les deux amants des Vertes années, un brutal plan d'ensemble noie les deux figures dans l'immensité d'un ciel vide, tout comme les dernières images montrent le personnage principal hagard dans une nuit sans fond. Reprenant ses droits aux hommes orgueilleux, la Nature de Changer de vie plonge, le temps d'une scène presque apocalyptique, le littoral dans un épais brouillard qui recouvre décor et silhouettes, et elle envoie la mer grignoter toute forme sur la plage.

L'un des plus grands mérites de l'ouvrage que Deleuze consacre à l'auteur de Surveiller et punir consiste à expliciter la dimension nietzschéenne de la réflexion politique de Foucault, qui l'amène à envisager le pouvoir non en termes abstraits ou sous la forme d'une ontologie mais uniquement en termes d'exercices de pouvoir se rapportant toujours à des forces ${ }^{14}$. Étant donné que c'est avant tout la « rencontre avec le pouvoir ${ }^{15}$ " qui intéresse Foucault dans les récits de vies infâmes, les réflexions deleuziennes aident à cerner plus en détail les dynamiques politiques au cœur des deux premiers films de Rocha - et en particulier leur différence qui tourne principalement autour de la distinction foucaldienne, inspirée de Blanchot, entre extériorité et dehors.

Deleuze explique :

Il faut distinguer l'extériorité et le dehors. [...] Le dehors concerne la force : si la force est toujours en rapport avec d'autres forces, les forces renvoient nécessairement à un dehors irréductible, qui n'a même plus de forme, fait de distances incomparables par lesquelles une force agit sur une autre ou est agie par une autre. C'est toujours du dehors qu'une force confère à d'autres, ou reçoit des 
autres, l'affectation variable qui n'existe qu'à telle distance ou sous tel rapport. Il y a donc un devenir des forces qui ne se confond pas avec l'histoire des formes, puisqu'il opère dans une autre dimension. Un dehors plus lointain que tout le monde extérieur et même que toute forme d'extériorité, dès lors infiniment plus proche. [...] Voilà ce que disent les forces du dehors : ce n'est jamais le composé, historique et stratifié, archéologique, qui se transforme, mais ce sont les forces composantes, quand elles entrent en rapport avec d'autres forces issues du dehors (stratégies). Le devenir, le changement, la mutation concernent les forces composantes, non les forces composées. [...] le dernier mot du pouvoir, c'est que la résistance est première ${ }^{16}$. et conditionnée par le régime. Les espaces périphériques sont finalement montrés comme autant de lieux qui malgré leur hétérogénéité obéissent aux mêmes lois que les espaces centraux de la capitale, à commencer par la banlieue agricole où des voyeurs passent leur journée à surveiller et traquer les couples amoureux - ce qui musèle la force contestataire de la disparate. Plusieurs personnages portent le même prénom, le film brouillant à plusieurs reprises leurs identités, et la compagne de la figure centrale, sous le coup d'un complexe social qui illustre une violente logique de lutte des classes, passe son temps à s'habiller et à se comporter comme la riche bourgeoise pour laquelle elle travaille - comme si elle cherchait coûte que coûte à se fondre dans l'imagemodèle de sa patronne. En tuant celle dont il est épris, Júlio rend sensibles à la fois sa détresse métaphysique et son horreur d'un monde où les lieux et les êtres sont réduits à des doubles, sans que puisse se produire ce processus de libération foucaldien grâce auquel la « reproduction du Même » devient « répétition du Différent ${ }^{17}$ ».

À l'inverse, le décor maritime de Changer de vie est tout entier tourné vers l'ailleurs, le lointain, c'est-à-dire qu'il appelle et construit un dehors cinématographique, géographique, et forcément politique, y compris en faisant muter le statut des (ex et futures ex) colonies. De " provinces extérieures », telles qu'elles ont été ou sont pensées par le régime, elles se transforment en lieu d'un renouveau possible du cinéma via l'hommage à Glauber Rocha. Grâce aux plans solaires et entêtants des vagues de l'Océan qui répètent leur mouvement, le Même est enfin Différent, la disparate laisse parler toute sa puissance, et les forces composantes du dehors peuvent enfin agir à l'échelle de l'ensemble du film sur les forces composées $d u$ dedans - ce qui bouleverse le statut du geste des Vertes années à Changer de vie. Si les deux œuvres accordent une grande importance à la beauté et à la précision des gestes artisanaux de celles et ceux qu'ils montrent, la première décrit une forme de corruption du geste, puisque ce sont la même main et le même couteau qui réparent les chaussures, façonnent une émouvante statuette en pomme de terre et mettent fin aux jours d'une jeune femme, alors que la seconde s'achève sur une réplique du personnage principal déclarant qu'il fait confiance «à ses bras ", donc à ses gestes et à sa capacité de travail, pour lui permettre de survivre et de continuer ainsi à résister.

Ce qui se joue entre les deux films, c'est la mise en mouvement des vies infâmes en tant que singularités. Pour Deleuze, il existe principalement trois types de singularités chez Foucault: les singularités de pouvoir, les singularités de résistance et les singularités sauvages. Ces dernières « restent suspendues au dehors, sans entrer dans des rapports ni même se laisser intégrer ${ }^{18}$ ". Les Vertes années fait entendre les cris romantiques d'une singularité sauvage qui s'époumone éternellement et risque de se perdre dans la nuit, Changer de vie trace la trajectoire de deux vies singulières, infâmes et sauvages, qui acceptent d'entrer dans des rapports de force et de résistance, réinventent la possibilité 
du changement et lancent une turbulence esthétique et politique qui anticipe déjà la venue d'une Révolution au nom fleuri.

\section{NOTES}

1. W. Shakespeare, Jules César, Acte I, scène 2, dans Tragédies, t. 1, trad. Jérôme Hankins, Paris, Gallimard, coll. « Pléiade », 2002, p. 473.

2. Le terme pinku eiga (littéralement « cinéma rose ») désigne le cinéma érotique japonais apparu dans les années soixante, souvent très contestataire.

3. M. Foucault, "La vie des hommes infâmes ", dans Philosophie - Anthologie, Texte ${ }^{\circ}$ 51, Paris, Gallimard, coll. « Folio Essais », 2004, p. 565.

4. Ibid., p. 567.

5. Par ce jeu autour de la guerre coloniale transformée en non dit obsessionnel et obsédant, il s'agit évidemment pour Rocha de tester les limites de la censure salazariste, qui interdisait toute référence explicite aux affrontements dans les colonies qu'il fallait désigner en tant que «provinces ultra-marines portugaises ».

6. Art. cit., p. 562.

7. Ibid., p. 580.

8. Ibid., p. 581.

9. Ibid., p. 565.

10. Ses deux recueils de poésie ne s'intitulent pas pour rien Poèmes quotidiens (1957) et Nouveaux poèmes quotidiens (1959).

11. Art. cit., p. 582.

12. G. Deleuze, Foucault, Chap. II, «Les stratégies ou le non-stratifié: la pensée du dehors (pouvoir) », Paris, Éditions de Minuit, coll. « Reprise », 2004 [1986], p. 72.

13. M. Foucault, Histoire de la folie à l'âge classique, Partie III, Chap. V, «Le cercle anthropologique », Paris, Gallimard, coll. « TEL », 1972, p. 655-656, passage consacré à la série de gravure Les Disparates, réalisée par Goya entre 1815 et 1823.

14. «Un exercice de pouvoir apparaît comme un affect, puisque la force se définit elle-même par son pouvoir d'affecter d'autres forces [...] et d'être affectée par d'autres forces. Inciter, susciter, produire [...] constituent des affects actifs, et être incité, être suscité, être déterminé à produire, avoir un effet « utile », des affects réactifs », op. cit., p. 78.

15. M. Foucault, « La vie des hommes infâmes », op. cit., p. 567.

16. G. Deleuze, Foucault, op. cit., p. 92-95.

17. «Le thème qui a toujours hanté Foucault, c'est celui du double. Mais le double n'est jamais une projection de l'intérieur, c'est au contraire une intériorisation du dehors. Ce n'est pas un dédoublement de l'Un, c'est un redoublement de l'Autre. Ce n'est pas une reproduction du Même, c'est une répétition du Différent. [...] c'est moi qui me vis comme un double de l'autre : je ne me rencontre pas à l'extérieur, je trouve l'autre en moi («il s'agit de montrer comment l'Autre, le lointain est aussi bien le plus Proche et le Même »). ", Ibid., Chap. III, «Les plissements, ou le dedans de la pensée (subjectivation) », p. 105.

18. Ibid., p. 125. 


\section{RÉSUMÉS}

La contextualisation de la filmographie de Paulo Rocha au sein du réseau d'influences et de collaborations qui la façonnent met en évidence un indéniable attrait pour le traitement cinématographique des vies infâmes, que l'analyse de ses deux premiers longs métrages, Les Vertes années et Changer de vie, permet d'exemplifier. Interrogées à l'aune de la pensée foucaldienne du dehors et de la notion de disparate langagière et visuelle, les deux œuvres dessinent un chemin vers la résistance esthétique et politique.

The contextualisation of Paulo Rocha's filmography, amongst the network of influences and collaborations that shape it, reveals a remarkable appeal for the lives of infamous men, that the portuguese director's two debut feature films, os Verdes anos and Mudar de vida, illustrate. Analyzed using Foucault's notions of linguistical and visual "disparate" and his concept of "outside", these two motion pictures draw a path towards aesthetic and political resistance.

\section{AUTEUR}

\section{GUILLAUME BOURGOIS}

Guillaume Bourgois est maître de conférences en études cinématographiques à l'université Grenoble Alpes. Auteur d'une thèse intitulée Poétique de la divergence : le cinéma d'Oliveira à la lumière de Pessoa (et de Godard), il travaille principalement sur le cinéma portugais, les films de Jean-Luc Godard, le cinéma américain et les séries télévisées. Il a publié des articles dans Trafic, les Cahiers du cinéma, Les Cahiers du Musée national d'Art Moderne ou L'Art du cinéma, ainsi qu'un essai sur L'étrange affaire Angélica de Manoel de Oliveira (De L'Incidence éditeur, 2013). Son prochain ouvrage intitulé Regards et Écoutes : Ray, Godard, Visconti \& friends est à paraître chez L'Harmattan et sera consacré à l'analyse de films. 